\title{
INSTANTONS ON CONIC 4-MANIFOLDS: FREDHOLM THEORY
}

\author{
Weiping Li and Shuguang Wang
}

\begin{abstract}
We study the self-duality operator on conic 4-manifolds. The self-duality operator can be identified as a regular singular operator in the sense of Brüning and Seeley, based on which we construct its parametrizations and closed extensions. We also compute the indexes.
\end{abstract}

\section{Introduction}

The main purpose of the paper is to initiate the investigation of gauge theory on a class of manifolds that have the simplest kind of singularity.

Let $X_{0}$ be a compact smooth manifold with boundary $Y$. The associated topological conical space $X$ is by definition the gluing $X_{0} \cup_{Y} N$ along $Y$, where $N=[0,1] \times Y /\{0\} \times Y$, i.e., one boundary $\{0\} \times Y$ collapsing into a single point $c$. Clearly $X$ is a compact topological space but the manifold structure at $c$ is singular. To best suit this type of singularity, we place the (close) conic metric of the form $d t^{2}+t^{2} g_{Y}$ on $N$ away from $c$, where $g_{Y}$ is any smooth Riemannian metric on $Y$.

Conical spaces and conic metrics arise naturally in many different contexts. In Algebraic Geometry, a conic singular complex surface is obtained by contracting a curve of negative self intersection in a smooth Kähler surface. The smooth Kähler metric descends to a conic metric on the complex quotient. The point of such a construction is that away from the singular point, the complex quotient is often topologically much simpler than the original Kähler surface. For example, contracting the sixteen $(-2)$-curves on a Kummer surface yields simply the quotient of a torus under the natural involution. It is well-known that negative curves are difficult to study since they are isolated and do not form any moduli. In Symplectic Geometry, for symplectic 4-manifolds with boundary a concave contact 3-manifold, the contact structure can be extended naturally into a symplectic form which is compatible with a conic metric on the end. A recent result of $\mathbf{J}$. Etnyre and K. Honda [8] shows that any concave

Received July 22, 2005.

2000 Mathematics Subject Classification. 57R57, 58G12, 53C05, 58G18, 58J20.

Key words and phrases. gauge theory, conic 4-manifold, self-duality operator, parametrization, closed extension, index. 
contact structure is fillable by infinitely many non-isomorphic symplectic manifolds, thus quite different from convex contact structures. In String Theory, there is a 6-dimensional conifold $X$ with base $S^{3} \times S^{2}$ introduced in GopakumarVafa [9]. The cone singularity can be resolved in two ways: either blow up the $S^{2}$ direction resulting in the bundle $\mathcal{O}(-1) \oplus \mathcal{O}(-1)$ over $S^{2}$, or deform along the $S^{3}$ direction resulting in the cotangent bundle $T^{*} S^{3}$. The conjectured duality in [9] says that the Gromov-Witten invariant of $\mathcal{O}(-1) \oplus \mathcal{O}(-1)$ is related to a Chern-Simons type knot invariant of $S^{3}$. A possible approach might be to study directly the conifold $X$ and relate the invariants to those on the resolutions.

The (close) conic metric on $X$ defined above is conformally equivalent to the open conic metric $d s^{2}+s^{2} g_{Y}$ and product metric $d s^{2}+g_{Y}$, where $1<s<+\infty$. However the geometry in the first case is considerably different and harder than the latter two cases. The main reason for such a disparity is due to the fact that the open conic and product metrics are both complete whereas the close conic metric is not. Thus the traditional analytic approach for complete metrics is no longer suitable and new phenomena do emerge in close conic metric geometry (see $[1,3,4,11])$.

In the aforementioned examples, it is necessary to work with close conic metrics, since open or product metrics can not be Kähler or symplectic. (Of course being Kähler or symplectic metrics is not conformally invariant.) The conic singularities and manifolds with boundary are closely related, and topological and geometric properties on manifolds with conic singularities may be viewed as a ramification of special boundary value problems with non-local boundary conditions. The conic singularity is interesting in its own right and was initially studied by Cheeger $[3,4]$.

In the current paper, we introduce Yang-Mills theory on the conic 4-manifolds. This leads to two major changes from the standard theory in smooth 4-manifolds (e.g. [5]). (1) Since the metric is not differentiable at the cone singular point, the slice condition and $d_{A}^{*}$ operator require a modification similar to [6]. (2) The functional-analytic framework and the Fredholm theory need different setups than $[5,6]$. In particular, the self-duality operator on conic manifolds may have many different closed extensions which are all Fredholm but with different domains and indices. Our main results can be summarized as follows.

Theorem. (1) The self-duality operator $d_{A}^{+} \oplus d_{A}^{*}$ on a conic 4 -manifold $X$ can be identified with a first order regular singular operator $P_{A}=\frac{\partial}{\partial t}+t^{-1} P_{0}+P_{1}$ in the neighborhood of the cone point. In particular it is of (weak) Fuchs type.

(2) Let $P_{A}^{i D}$ be the restriction of $P_{A, \max }$ to the ideal Dirichlet boundary condition. The operator $P_{A}^{i D}$ is closed and Fredholm.

(3) The index of $P_{A}^{i D}$ is given by

$$
\int_{X} \tilde{c}_{2}(\operatorname{ad} P)-\frac{1}{2}\left(\eta_{P_{0}}+m_{1}\right)+\sum_{k \geq 1} \frac{2^{-2 k}}{2 k(2 k+1)} \operatorname{Res}_{1} \eta_{P_{0}}(2 k),
$$


where $\eta_{P_{0}}(z)$ is the eta function of the operator $P_{0}$ and $m_{1}$ is the multiplicity of eigenvalue $1 / 4$ of the Laplacian operator $\Delta^{1}$ on coclosed $\Omega^{1}(Y)$ with the metric $g_{Y}$.

The paper is organized as follows. Section 2 gives a basic set-up and shows part (1) of the Theorem in two lemmas. The parametric construction, following from the method in $[1,2]$ (or the more general treatment in [11]), is given in section 3. Then we prove part (2) in Theorem 3.5. Unlike the smooth manifold case, there are different closed extensions of the operator $P_{A}$. By applying the results in $[1,2]$, we obtain the index formula part (3) in the last section.

\section{A basic set-up}

Let $X$ be a closed, oriented conical 4-manifold with a cone point $c$. (The analysis can be naturally extended to the case of a finite number of cone points.) Let $N$ be a neighborhood of $c$ in $X, N=(0, \varepsilon] \times Y$, where the 3-manifold $Y$ has a metric $g_{Y}$. Note that the 4-dimensional Yang-Mills theory is conformally unchanged. Thus up to conformal changes, $N$ can be equipped with the metric

$$
d t^{2}+t^{2} g_{Y}
$$

The metric satisfies the conformally conic manifold definition 1.1.1 of [11] (with $h=1, c=0>-1$ and any $\delta$ ). The manifold is a product near the singularity. Our point of view is to transfer the geometric singularity into a singularity of a geometric differential operator on a manifold with boundary. The natural differential operators involving the conic metric are differential operators of regularly singular operators $[1,2]$ or Fuchs type operators [11].

Let $P$ be an $S U(2)$-principal bundle over $X$. Let $\mathcal{A}(P)$ be the space of $S U(2)$-connections on the principal bundle $P$, and $\mathcal{G}(P)$ be the automorphism group of $P$. Note that $\left.\mathcal{A}(P)\right|_{N}$ is an affine space modeled on $\Omega^{1}\left(N,\left.\operatorname{ad} P\right|_{N}\right)$. We can naturally identify $\Omega^{1}\left(N,\left.\operatorname{ad} P\right|_{N}\right)$ with $\Omega^{1}\left(Y, \operatorname{ad} P_{Y}\right) \oplus \Omega^{0}\left(Y,\left.\operatorname{ad} P\right|_{Y}\right)$. More generally and in order to perform further calculations, we give an identification between $p$-forms on $N$ and $p$-forms, $(p-1)$-forms on $Y$ :

$$
\begin{gathered}
f_{p}: \Omega^{p}\left(Y, \operatorname{ad} P_{Y}\right) \oplus \Omega^{p-1}\left(Y,\left.\operatorname{ad} P\right|_{Y}\right) \rightarrow \Omega^{p}\left(N,\left.\operatorname{ad} P\right|_{N}\right) \\
\left(w_{p}, w_{p-1}\right) \mapsto t^{p-3 / 2}\left(\pi^{*} w_{p}+t^{-1} \pi^{*} w_{p-1} \wedge d t\right),
\end{gathered}
$$

where $\pi: N=(0, \varepsilon) \times Y \rightarrow Y$ is the projection on the second factor. Note that one has

$$
\begin{aligned}
& \left\|f_{p}\left(w_{p}, w_{p-1}\right)\right\|_{L^{2}\left(\Omega^{p}\left(N,\left.\operatorname{ad} P\right|_{N}\right)\right)}^{2} \\
= & \int_{0}^{\varepsilon}\left\{\left\|w_{p}\right\|_{L^{2}\left(\Omega^{p}\left(Y, \operatorname{ad} P_{Y}\right)\right)}^{2}+\left\|w_{p-1}\right\|_{L^{2}\left(\Omega^{p-1}\left(Y,\left.\operatorname{ad} P\right|_{Y}\right)\right)}^{2}\right\} d t .
\end{aligned}
$$


With respect to the metric $g_{N}=d t^{2}+t^{2} g_{Y}$,

$$
\begin{aligned}
& *_{g_{N}} f_{p}\left(w_{p}, w_{p-1}\right) \\
= & *_{g_{N}}\left(t^{p-3 / 2}\left(\pi^{*} w_{p}+t^{-1} \pi^{*} w_{p-1} \wedge d t\right)\right) \\
= & t^{3 / 2-p} \pi^{*}\left(*_{g_{Y}} w_{p}\right) \wedge d t+t^{5 / 2-p} \pi^{*}\left((-1)^{4-p} *_{g_{Y}} w_{p-1}\right) \\
= & t^{(4-p)-3 / 2}\left(\pi^{*}\left((-1)^{4-p} *_{g_{Y}} w_{p-1}\right)+t^{-1} \pi^{*}\left(*_{g_{Y}} w_{p}\right) \wedge d t\right) \\
= & f_{4-p}\left((-1)^{4-p} *_{g_{Y}} w_{p-1}, *_{g_{Y}} w_{p}\right) .
\end{aligned}
$$

Hence we obtain the Hodge-star operator $*_{g_{N}}$ acting on the identification (1):

$$
*_{g_{N}} f_{p}\left(w_{p}, w_{p-1}\right)=f_{4-p}\left((-1)^{4-p} *_{g_{Y}} w_{p-1}, *_{g_{Y}} w_{p}\right) \text {. }
$$

The restricted gauge group over $N$ is denoted by $\left.\mathcal{G}(P)\right|_{N}$. For any connection $\left.A \in \mathcal{A}(P)\right|_{N}$, there is a (temporal) gauge transformation in $\left.\mathcal{G}(P)\right|_{N}$ under which $A$ does not contain the covariant derivative in $t$-direction, i.e., $A=a(t)$, where each $a(t)$ is a connection on $Y$ and $t \in(0, \varepsilon)$. Note that the connection $a(t)$ may not be defined at $t=0$ corresponding the cone point in $X$. The covariant derivative $d_{A}: \Omega^{0}(N, \operatorname{ad} P \mid N) \rightarrow \Omega^{1}\left(N,\left.\operatorname{ad} P\right|_{N}\right)$ can be transformed over to

$$
d_{A}: \Omega^{0}\left(Y,\left.\operatorname{ad} P\right|_{Y}\right) \rightarrow \Omega^{1}\left(Y,\left.\operatorname{ad} P\right|_{Y}\right) \oplus \Omega^{0}\left(Y,\left.\operatorname{ad} P\right|_{Y}\right) .
$$

We need to calculate $d_{A}^{*}: \Omega^{1}\left(N,\left.\operatorname{ad} P\right|_{N}\right) \rightarrow \Omega^{0}(N, \operatorname{ad} P \mid N)$ through the identification (1).

Lemma 2.1. The operator $d_{A}^{*}$ can be identified through (1) with the operator:

$$
\begin{gathered}
\Omega^{1}\left(Y,\left.\operatorname{ad} P\right|_{Y}\right) \oplus \Omega^{0}\left(Y,\left.\operatorname{ad} P\right|_{Y}\right) \rightarrow \Omega^{0}\left(Y,\left.\operatorname{ad} P\right|_{Y}\right) \\
(\alpha, \phi) \mapsto \frac{\partial \phi}{\partial t}+t^{-1} d_{a}^{*_{g_{Y}}} \alpha+\frac{3}{2} t^{-1} \phi .
\end{gathered}
$$

Proof. The proof is given by a direct calculation as follows.

$$
\begin{aligned}
d_{A}^{*} f_{1}(\alpha, \phi)= & (-1)^{(p-1) n+1} *_{g_{N}} d_{A}\left(*_{g_{N}} f_{1}(\alpha, \phi)\right) \\
= & (-1) *_{g_{N}} d_{A}\left(f_{3}\left(-*_{g_{Y}} \phi, *_{g_{Y}} \alpha\right)\right) \\
= & (-1) *_{g_{N}} d_{A}\left(t^{3 / 2}\left(-\pi^{*}\left(*_{g_{Y}} \phi\right)+t^{1 / 2} \pi^{*}\left(*_{g_{Y}} \alpha\right) \wedge d t\right)\right. \\
= & *_{g_{N}}\left(\frac{3}{2} t^{1 / 2} \pi^{*}\left(*_{g_{Y}} \phi\right) \wedge d t\right. \\
& +t^{3 / 2} \pi^{*}\left(*_{g_{Y}} \frac{\partial \phi}{\partial t} \wedge d t-t^{1 / 2} \pi^{*}\left(d_{a} *_{g_{Y}} \alpha\right) \wedge d t\right) \\
= & *_{g_{N}} f_{4}\left(0, *_{g_{Y}} \frac{\partial \phi}{\partial t}-t^{-1} d_{a} *_{g_{Y}} \alpha+t^{-1} \frac{3}{2} *_{g_{Y}} \phi\right) \\
= & f_{0}\left(\frac{\partial \phi}{\partial t}+t^{-1} d_{a}^{*} g_{Y} \alpha+\frac{3}{2} t^{-1} \phi, 0\right) .
\end{aligned}
$$

The identity follows from (1) and (2), since the connection $A=\left\{a_{t}\right\}_{t \in(0, \varepsilon)}$ has no $d t$-component. 
With respect to the conic metric $g_{N}$ on $N$, one has

$$
d_{A}^{+}: \Omega^{1}\left(N,\left.\operatorname{ad} P\right|_{N}\right) \rightarrow \Omega_{+}^{2}\left(N,\left.\operatorname{ad} P\right|_{N}\right) .
$$

Through the identifications (1) and (2), we have the following result.

Lemma 2.2. The operator $d_{A}^{+}$can be identified with the operator

$$
\begin{gathered}
\Omega^{1}\left(Y,\left.\operatorname{ad} P\right|_{Y}\right) \oplus \Omega^{0}\left(Y,\left.\operatorname{ad} P\right|_{Y}\right) \rightarrow \Omega^{1}\left(Y,\left.\operatorname{ad} P\right|_{Y}\right) \\
(\alpha, \phi) \mapsto \frac{\partial \alpha}{\partial t}+t^{-1}\left(*_{g_{Y}} d_{a} \alpha+d_{a} \phi-\frac{1}{2} \alpha\right) .
\end{gathered}
$$

Proof. We calculate the corresponding $d_{A}$ first.

$$
\begin{aligned}
d_{A} f_{1}(\alpha, \phi)= & d_{A}\left(t^{-1 / 2}\left(\pi^{*} \alpha+t^{-1} \pi^{*} \phi \wedge d t\right)\right) \\
= & t^{-1 / 2} \pi^{*} d_{a} \alpha-\frac{1}{2} t^{-3 / 2} \pi^{*} \alpha \wedge d t \\
& +t^{-1 / 2} \pi^{*}\left(\frac{\partial \alpha}{\partial t}\right) \wedge d t+t^{-2 / 3} \pi^{*}\left(d_{a} \phi\right) \wedge d t \\
= & t^{2-3 / 2}\left(t^{-1} \pi^{*}\left(d_{a} \alpha\right)+t^{-1} \pi^{*}\left(\frac{\partial \alpha}{\partial t}+t^{-1} d_{a} \phi-\frac{1}{2} t^{-1} \alpha\right) \wedge d t\right) \\
= & f_{2}\left(t^{-1} d_{\alpha} \alpha, \frac{\partial \alpha}{\partial t}+t^{-1} d_{a} \phi-\frac{1}{2} t^{-1} \alpha\right) .
\end{aligned}
$$

Now by the definition of $d_{A}^{+}=\frac{1+*_{g_{N}}}{2} d_{A}$ and the above equality, we have $d_{A}^{+} f_{1}(\alpha, \phi)=\frac{1}{2} f_{2}\left(w_{2}, w_{1}\right)$ by $(2)$, where

$$
\begin{aligned}
& w_{2}=t^{-1} d_{a} \alpha+*_{g_{Y}} \frac{\partial \alpha}{\partial t}+t^{-1} *_{g_{Y}} d_{a} \phi-\frac{1}{2} t^{-1} *_{g_{Y}} \alpha, \\
& w_{1}=\frac{\partial \alpha}{\partial t}+t^{-1} d_{a} \phi-\frac{1}{2} t^{-1} \alpha+t^{-1} *_{g_{Y}} d_{a} \alpha .
\end{aligned}
$$

The components $w_{2}$ and $w_{1}$ are identified by the Hodge operator $*_{g_{Y}}$ on $Y$. Therefore $d_{A}^{+}$can be identified with $w_{1}$ through (1) and (2).

Remark. As in the usual instanton theory on smooth 4-manifolds, we need to use $d_{A}^{*}$ to build a slice of the configuration space and to get the elliptic operator $d_{A}^{+} \oplus d_{A}^{*}$. We will follow the method used in $[1,2]$ to build a parametrix for the self-duality operator with conic singularity. For the singular operator $d_{A}^{+} \oplus d_{A}^{*}$, the construction of parameterization can be constructed from the general method in [11].

Thus the self-duality operator $d_{A}^{+} \oplus d_{A}^{*}$ restricted on $N$ can be identified with

$$
P_{A}: \Omega^{1}\left(Y,\left.\operatorname{ad} P\right|_{Y}\right) \oplus \Omega^{0}\left(Y,\left.\operatorname{ad} P\right|_{Y}\right) \rightarrow \Omega^{1}\left(Y,\left.\operatorname{ad} P\right|_{Y}\right) \oplus \Omega^{0}\left(Y,\left.\operatorname{ad} P\right|_{Y}\right)
$$

by Lemma 2.1 and Lemma 2.2 through the $L^{2}$-isometry. So we have:

$$
P_{A}(\alpha, \phi)=\left(\begin{array}{cc}
\frac{\partial}{\partial t}+\frac{1}{t} *_{Y} d_{a}-\frac{1}{2} & t^{-1} d_{a} \\
\frac{1}{t} d_{a}^{*} Y & \frac{\partial}{\partial t}+\frac{3}{2} t^{-1}
\end{array}\right) \cdot\left(\begin{array}{c}
\alpha \\
\phi
\end{array}\right) .
$$

As an operator,

$$
P_{A}=\frac{\partial}{\partial t}+\frac{1}{t}\left(\begin{array}{cc}
*_{Y} d_{a}-\frac{1}{2} & d_{a} \\
d_{a}^{*} Y & \frac{3}{2}
\end{array}\right)
$$


Let us recall Definition 1.1.2 of [11] about operators of Fuchs type.

Definition 2.3. 1. Let $E, F$ be Hermitian vector bundles over $X$. We denote by $\operatorname{Diff}^{\mu}(E, F)$ the set of differential operators of order $\leq \mu$ acting from sections in $E$ to sections in $F$.

2. Denote by $t$ the operator of multiplication by $t$ and put $D=-t \frac{\partial}{\partial t}$. A differential operator of Fuchs type of order $\mu \in \mathbf{Z}_{+}$and weight $\nu \geq 0$ is an operator $P \in \operatorname{Diff}^{\mu}(E, F)$ such that

$$
\left.P\right|_{N}=t^{-\nu} \sum_{k=0}^{\mu} B_{k} D^{k},
$$

with $B_{k} \in C^{\infty}\left((0, \varepsilon), \operatorname{Diff}^{\mu-k}\left(\left.E\right|_{N},\left.F\right|_{N}\right)\right) \cap C\left([0, \varepsilon), \operatorname{Diff}^{\mu-k}\left(\left.E\right|_{N},\left.F\right|_{N}\right)\right)$.

3. $P$ is of weak Fuchs type if $B_{k} \in C^{\infty}\left((0, \varepsilon), \operatorname{Diff}^{\mu-k}\left(\left.E\right|_{N},\left.F\right|_{N}\right)\right)$ only.

Lemma 2.4. The operator $d_{A}^{+} \oplus d_{A}^{*}: \Omega^{1}(X, \operatorname{ad} P) \rightarrow \Omega^{0} \oplus \Omega_{+}^{2}(X, \operatorname{ad} P)$ is a differential operator of weak Fuchs type of order 1 and weight 1 .

Proof. Through the identification with the operator $P_{A}$ on the conic neighborhood $N$, it is sufficient to note that $P_{A}$ can be written as

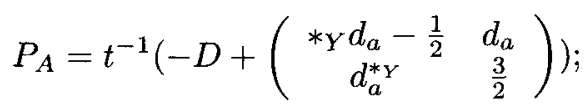

so in the notations of Definition 2.3,

$$
B_{1}=I d \text { and } B_{0}(t)=\left(\begin{array}{cc}
*_{Y} d_{a}-\frac{1}{2} & d_{a} \\
d_{a}^{* Y} & \frac{3}{2}
\end{array}\right)
$$

are both smooth for $t \in(0, \varepsilon)$. Hence $d_{A}^{+} \oplus d_{A}^{*}$ is of weak Fuchs type.

We obtain weak Fuchs type only since $a(t)$ and therefore $B_{0}(t)$ are undefined at $t=0$ in general.

Note that the operator $d_{A}^{+}$alone cannot be a differential operator of Fuchs type and the techniques and estimates in $[1,11]$ cannot be applied. To remedy this, we need to include $d_{A}^{*}$, i.e., consider the self-duality operator as a whole.

\section{A parametrix construction}

In this section, we construct parametrices for our first order self-duality operator of Fuchs type near the boundary (the neighborhood of the cone singularity). The important feature of the construction is that the space of closed extensions is finite dimensional and closed extensions are Fredholm operators for the operator of Fuchs type on a compact manifold with conic singularities.

To get the parametrices, we need some kind of ellipticity for the self-duality operator. The operator is certainly elliptic in the interior of $X$. In addition some condition must be imposed near the tip $t \rightarrow 0$. We follow Lesch's approach [11] here. So we need now to strengthen our requirement for the connection $A=a(t)$ near $t=0$ : 
Assumption. There is a smooth connection $a_{0}$ on $P_{Y}$ such that $\sup _{Y} \mid a(t)$ $-a_{0} \mid \leq C t^{1+\nu}$ for some number $\nu>0$.

This assumption will be used throughout the rest of our paper. With this assumption, $B_{0}(0)$ exists and $P_{A}=t^{-1}\left(B_{0}+D\right)$ is a first order differential operator of Fuchs type as in Definition 2.3. Moreover we can express our operator as

where

$$
P_{A}=\frac{\partial}{\partial t}+t^{-1} P_{0}+P_{1}
$$

$$
P_{0}=\left(\begin{array}{cc}
*_{Y} d_{a_{0}}-\frac{1}{2} & d_{a_{0}} \\
d_{a_{0}}^{*_{Y}} & \frac{3}{2}
\end{array}\right), P_{1}=\frac{1}{t}\left(\begin{array}{cc}
*_{g Y}\left(a(t)-a_{0}\right) & a(t)-a_{0} \\
*_{g_{Y}}\left(a_{0}-a(t)\right) *_{g_{Y}} & 0
\end{array}\right)
$$

are smooth operators away from $t=0$ and continuous up to $t=0$. Also note that $P_{1}$ is a compact zero-th order operator.

As for ellipticity near $t=0$, we observe that

$$
\sigma_{X}^{1,1}\left(P_{A}\right)(z):=\sum_{k=0}^{\mu} B_{k}(0) z^{k}=B_{0}(0)+z
$$

is a parameter dependent elliptic family of differential operators with parameter $z \in i \mathbf{R}$. Hence $P_{A}$ is an elliptic operator of Fuchs type in the sense of [11]. There is a general method in [11] to get the parametrix construction. We would like to work out explicitly for the self-duality operator with cone singularity in order to understand better on the behaviors from analytic point of view.

Digression. Existence of a limiting connection at the conic singularity.

The assumption above is satisfied in our intended applications that will include orbifold singularities, blowing down negative curves etc. To illustrate this and also more specifically the difference between a close conic metric and an open conic metric/cube metric that was alluded in the Introduction, we pause to digest the simple case of $\mathbf{R}^{4}$ with the standard Euclidean metric. It is well known that a charge $1, S U(2)$-instanton, with center at 0 , is

$$
A=\operatorname{Im}\left\{\frac{\bar{x} d x}{1+x^{2}}\right\},
$$

where $x \in \mathbf{R}^{4}=\mathbf{H}$ is viewed as quaternion. Its curvature is $F=\frac{d x \wedge d \bar{x}}{\left(1+|x|^{2}\right)^{2}}$ with density $|F|=\left(1+|x|^{2}\right)^{-2}$. We view the origin as the trivial cone point with base $S^{3}$, and we can rewrite the connection in terms of the generalized polar coordinates $(r, \theta)$ as

$$
A=\frac{r^{2}}{1+r^{2}} \operatorname{Im}\left(\theta^{-1} d \theta\right)
$$

where $\theta \in S^{3}$ is viewed as a unit quaternion. This means that $a(r) \rightarrow 0$ here as we approach the cone tip $r=0$, i.e., the assumption above is satisfied. Note that $A$ is not flat near $0:|F|$ approaches 1 as $r \rightarrow 0$. This contrasts with the open cone at $\infty$ of $\mathbf{R}^{4}$, where $A$ is asymptotically flat. (Incidentally this 
suggests that some kind of gluing procedure is possible between close and open conical instantons). Now transform $\mathbf{R}^{4} \backslash\{0\}$ into the cylinder $(-\infty, \infty) \times S^{3}$ via the map $(r, \theta) \mapsto(\ln r, \theta)$. Of course, the model connection $A$ is still not asymptotically flat near the end $t=\ln r \rightarrow-\infty$ under the equivalent metric $d r^{2}+r^{2} d \theta^{2}=e^{2 t}\left(d t^{2}+d \theta^{2}\right)$. However under the tube metric $d t^{2}+d \theta^{2}$, which is conformally equivalent to the Euclidean metric, $A$ is asymptotically flat near $-\infty$, since the curvature density under the tube metric is $|F|=\frac{4}{\cosh ^{2}{ }^{2}}$, which goes to 0 as $t \rightarrow-\infty$. This simple picture explains why we can have exponential decays for curvature of instantons under tube metrics but we can not do so under close conic metrics. Moreover it indicates that conformal metrics can lead to quite different geometry.

Let us return to $P_{A}$. The operator $P_{A}$, acting on $L^{2}((0, \varepsilon), H)$ with domain $C_{0}^{\infty}\left((0, \varepsilon), H_{P}\right)$, amounts to integration of a first order ordinary differential equation, where $H=L_{k}^{2}\left(\Omega^{1}\left(Y,\left.\operatorname{ad} P\right|_{Y}\right) \oplus \Omega^{0}\left(Y,\left.\operatorname{ad} P\right|_{Y}\right)\right)$ and $H_{P}$ is the common domain of the family of self-adjoint operators $P_{0}+t P_{1}, t \in(0, \varepsilon]$, in $L_{k+1}^{2}\left(\Omega^{1}\left(Y,\left.\operatorname{ad} P\right|_{Y}\right) \oplus \Omega^{0}\left(Y,\left.\operatorname{ad} P\right|_{Y}\right)\right)$. Hence we have the following operator norm inequality:

$$
\left\|P_{1}\left(\left|P_{0}\right|+1\right)^{-1}\right\|+\left\|\left(P_{0}+1\right)^{-1} P_{1}\right\| \leq C,
$$

where the estimate is uniformly in $(0, \varepsilon]$ from our assumption on $a_{t}$. This is, in fact, a first order regular singular operator $P_{A}$ from $[1,2,3,4]$.

For $f \in L^{2}(0, \infty)$, define two integral operators

$$
\begin{gathered}
P_{0, s} f(t)=\int_{0}^{t}\left(\frac{x}{t}\right)^{s} f(x) d x, \quad s>-\frac{1}{2} \\
P_{1, s} f(t)=\int_{1}^{t}\left(\frac{x}{t}\right)^{s} f(x) d x, \quad s<\frac{1}{2}
\end{gathered}
$$

On $L^{2}(0, \infty)$, these two integral operators satisfy the identity

$$
\left(\frac{\partial}{\partial t}+t^{-1} s\right) P_{0, s}=\left(\frac{\partial}{\partial t}+t^{-1} s\right) P_{1, s}=I d .
$$

Our parameterizations are constructed from the combinations of $P_{0, s}$ and $P_{1, s}$ near $t=0$.

Lemma 3.1. For $f \in L^{2}(0, \infty)$ and $t$ sufficiently small, the following estimates hold.

(1) $\left|P_{0, s} f(t)\right| \leq \sqrt{\frac{t}{|2 s+1|}} \cdot\|f\|_{L^{2}(0, t)}$ for $s>-1 / 2$;

$$
\left|P_{1, s} f(t)\right| \leq \begin{cases}\sqrt{\frac{t}{|2 s+1|}} \cdot\|f\|_{L^{2}} & s<-1 / 2 \\ \sqrt{t|\ln t|}\|f\|_{L^{2}(0, \delta)}+\sqrt{t|\ln \delta|}\|f\|_{L^{2}} & s=-1 / 2,0<\delta<1 .\end{cases}
$$

(2) for $|s|<1 / 2$,

$$
\left|P_{1, s} f(t)+t^{-s} \int_{0}^{1} x^{s} f(x) d x\right| \leq \sqrt{\frac{t}{2 s+1}}\|f\|_{L^{2}} .
$$


(3) for $0<\varepsilon \leq 1$,

$$
\begin{aligned}
& \left\|P_{0, s}\right\|+\left\|P_{1,-s}\right\| \leq C_{1}(s, \varepsilon)(|s|+1)^{-1}, \quad s>-\frac{1}{2}, \\
& \left\|P_{1, s}\right\|+\left\|P_{0,-s}\right\| \leq C_{2}(s, \varepsilon)(|s|+1)^{-1}, \quad s<1 / 2,
\end{aligned}
$$

where $\lim _{\varepsilon \rightarrow 0} C_{i}(s, \varepsilon)=0, i=1,2$, and uniformly for $|s| \geq 2$.

Proof. (1) and (2) follow from direct calculations. (3) follows from $\left(P_{0, s} t^{r}\right)^{*}=$ $-t^{r} P_{1, s}$, where $P_{0, s} t^{r}$ is bounded in $L^{2}(0, \varepsilon)$, and from the standard estimates for integral operators (see [1] Lemma 2.1 and Lemma 2.2 and $[7,12]$ ).

The maximum extension $P_{A, \max }$ of $P_{A}$ has domain

$$
\mathcal{D}\left(P_{A, \max }\right)=\left\{u \in L^{2}\left(\Omega^{1} \oplus \Omega^{0}\left(Y,\left.\operatorname{ad} P\right|_{Y}\right)\right) \mid P_{A} u \in L^{2}\left(\Omega^{1} \oplus \Omega^{0}\left(Y,\left.\operatorname{ad} P\right|_{Y}\right)\right)\right\} .
$$

A kind of ideal Dirichlet boundary condition for $P_{A}$ is defined to be

$$
\mathcal{D}^{i D}\left(P_{A}\right)=\left\{u \in \mathcal{D}\left(P_{A, \max }\right) \mid\|u(t)\|_{H}=o(1) \quad \text { as } t \rightarrow 0\right\},
$$

Denote by $P_{A}^{i D}$ the restriction of $P_{A, \max }$ to the domain $\mathcal{D}^{i D}\left(P_{A}\right)$. The boundary parametrix $Q^{i D}$ is given by

$$
Q^{i D}=\oplus_{s \in \operatorname{Spec} P_{0}, s \geq 0} P_{0, s} \oplus \oplus_{s \in \operatorname{Spec} P_{0}, s<0} P_{1, s},
$$

with each term $P_{i, s}(i=0,1)$ in the appropriate eigenspace of $P_{0}$.

Lemma 3.2. If $\psi \in C_{0}^{\infty}(-1,1)$, then $\psi Q^{i D}$ maps $L^{2}((0,1), H)$ into $\mathcal{D}^{i D}\left(P_{A}\right)$.

Proof. By Lemma $3.1(1)$ and $(2), f(t)=\oplus_{s \in \operatorname{Spec} P_{0}} f_{s}(t)$ has an unique expression with $f_{s}(t)$ projection on the eigenspace of $P_{0}$ for the eigenvalue $s$.

$$
\left\|\oplus_{s \geq 0} P_{0, s} f_{s}(t)\right\|_{H}^{2} \leq\left\|t \cdot \sum_{s \geq 0} \frac{s}{2 s+1} \int_{0}^{t}\left|f_{s}(x)\right|^{2} d x\right\|_{H} \leq C t \int_{0}^{t}\|f(t)\|_{H}^{2} d t .
$$

Similarly, we have

$$
\begin{aligned}
\left\|\oplus_{s<0} P_{1, s} f_{s}(t)\right\|_{H}^{2} \leq & C\left(\sum_{-1 / 2<s<0} t^{-2 s}\left\|f_{s}\right\|_{L^{2}}^{2}+t|\ln t|\left\|f_{-1 / 2}\right\|_{L^{2}}^{2}\right. \\
& \left.+t \sum_{s<-1 / 2} \frac{|s|}{|2 s+1|}\left\|f_{s}\right\|_{L^{2}}^{2}\right)=o(1)\|f\|_{L^{2}}^{2}
\end{aligned}
$$

as $t \rightarrow 0$. So $Q^{i D} f(t)=o(1)$ as $t \rightarrow 0$. One has the following:

$$
\begin{aligned}
P_{A}\left(\psi Q^{i D} f\right) & =\psi P_{A} Q^{i D} f+\psi^{\prime} Q^{i D} f \\
& =\psi\left(\frac{\partial}{\partial t}+t^{-1} P_{0}+P_{1}\right)\left(\oplus_{s \in \operatorname{Spec} P_{0}} f_{s}(t)\right)+\psi^{\prime} Q^{i D} f \\
& =\psi\left(\sum_{s \in \operatorname{Spec} P_{0}}\left(\frac{\partial}{\partial t}+t^{-1} P_{0}\right) f_{s}\right)+\psi P_{1} Q^{i D} f+\psi^{\prime} Q^{i D} f \\
& =\psi f+\psi P_{1} Q^{i D} f+\psi^{\prime} Q^{i D} f
\end{aligned}
$$


where $f_{s}(t)=P_{0, s} f(t)$ if $s \geq 0$, and $P_{1, s} f(t)$ if $s<0$. The last equality follows from (6). It now suffices to estimate

$$
\begin{aligned}
\left\|\left(\left|P_{0}\right|+1\right) Q^{i D} f\right\|_{H}^{2} & =\sum_{s \geq 0}(|s|+1)^{2}\left\|P_{0, s} f_{s}\right\|_{H}^{2}+\sum_{s<0}(|s|+1)^{2}\left\|P_{1, s} f_{s}\right\|_{H}^{2} \\
& \leq \sum_{s \geq 0} C_{1}(s, \varepsilon)^{2}\left\|f_{s}\right\|_{L^{2}}^{2}+\sum_{s<0} C_{2}(s, \varepsilon)^{2}\left\|f_{s}\right\|_{L^{2}}^{2} \\
& \leq \max \left\{C_{1}(s, \varepsilon)^{2}, C_{2}(s, \varepsilon)^{2}\right\}\|f\|^{2}
\end{aligned}
$$

by Lemma 3.1 (3). Thus $\psi Q^{i D}$ maps into the correct domain.

Lemma 3.3. If $u \in \mathcal{D}^{i D}\left(P_{A}\right)$ and $u(t) \equiv 0$ for $t \geq 1 / 2$, then $Q^{i D} P_{A} u=$ $u+\left(Q^{i D} P_{1}\right) u$.

Proof. Let $\left\{e_{s}\right\}_{s \in \operatorname{Spec} P_{0}}$ be an orthonormal basis in $H$ with $P_{0} e_{s}=s e_{s}$. Define $v(t)=\left(\frac{\partial}{\partial t}+t^{-1} P_{0}\right) u(t)$. So one has $v(t)=P_{A} u(t)-P_{1} u(t)$. Thus $v_{s}(t)=$ $\left\langle v(t), e_{s}\right\rangle$ can be calculated by

$$
\begin{aligned}
v_{s}(t) & =\left\langle\left(\frac{\partial}{\partial t}+t^{-1} P_{0}\right) u(t), e_{s}\right\rangle \\
& =\left\langle u^{\prime}(t)+t^{-1} P_{0} u(t), e_{s}\right\rangle \\
& =u_{s}^{\prime}(t)+t^{-1} s u_{s}(t), \quad s \in \operatorname{Spec} P_{0} .
\end{aligned}
$$

So $v_{s} \in L^{2}(0,1)$. Since $u_{s}(1)=0$ for all $s \in \operatorname{Spec} P_{0}$, for $s<1 / 2$,

$$
\begin{aligned}
P_{1, s} v_{s}(t) & =P_{1, s}\left(u_{s}^{\prime}(t)+t^{-1} s u_{s}(t)\right) \\
& =\int_{1}^{t}\left(\frac{x}{t}\right)^{s}\left(u_{s}^{\prime}(x)+x^{-1} s u_{s}(x)\right) d x \\
& =u_{s}(t),
\end{aligned}
$$

from the integration by parts; for $s \geq 0$, we use $P_{0, s}$ on $v_{s}(t)$ :

$$
\begin{aligned}
P_{0, s} v_{s}(t) & =\int_{0}^{t}\left(\frac{x}{t}\right)^{s}\left(u_{s}^{\prime}(x)+x^{-1} s u_{s}(x)\right) d x \\
& =t^{-s} \lim _{\delta \rightarrow 0} \int_{\delta}^{t} x^{s} u_{s}^{\prime}(x) d x+t^{-s} \int_{0}^{t} s x^{s-1} u_{s}(x) d x \\
& =u_{s}(t)-t^{-s} \lim _{\delta \rightarrow 0} \delta^{s} u_{s}(\delta) .
\end{aligned}
$$

For $s \geq 0$ and $v \in L^{1}$, we have $\left|P_{0, s} v(t)\right| \leq \int_{0}^{t}|v(x)| d x=o(1)$, and $u_{s}(t)=o(1)$ as $u \in \mathcal{D}^{i D}\left(P_{A}\right)$ for $t \rightarrow 0$ sufficiently small. Hence $P_{0, s} v(t)-u_{s}(t)=o(1)(=$ $\left.-t^{-s} \lim _{\delta \rightarrow 0} \delta^{s} u_{s}(\delta)\right)$ as $t \rightarrow 0$. Therefore $\left.\lim _{\delta \rightarrow 0} \delta^{s} u_{s}(\delta)\right)=0$ for $s>0$, i.e., $u_{s}(t)=P_{0, s} v_{s}(t)$ for $s \geq 0$. This completes the proof with the right domain.

Lemma 3.4. There is a positive $\varepsilon \leq 1$ such that for $\phi, \psi \in C_{0}^{\infty}(-\varepsilon, \varepsilon)$ with $\psi \phi=\phi$ and $u \in \mathcal{D}^{i D}\left(P_{A}\right)$,

$$
\phi u=V \psi Q^{i D} P_{A} \phi u
$$


for some bounded operator $V \in L^{2}((0,1), H)$. Moreover $\left\|\phi\left(\left|P_{0}\right|+1\right) u\right\|_{H} \leq$ $C\left\|P_{A} \phi u\right\|_{H}$.

Proof. Choose $\chi \in C_{0}^{\infty}(-\varepsilon, \varepsilon)$ with $\chi \psi=\psi$. Since $\phi u \in \mathcal{D}^{i D}\left(P_{A}\right)$, we have $f=P_{A}(\phi u) \in \mathcal{D}^{i D}\left(P_{A}\right)$ with $f(t) \equiv 0$ for $t \geq 1 / 2$. By Lemma 3.3,

$$
f=\phi u=Q^{i D} P_{A} f-Q^{i D} P_{1} f=Q^{i D} P_{A} \phi u-Q^{i D} P_{1} \phi u
$$

By the choices of smooth functions $\psi \phi=\phi, \chi \psi=\psi$ and $\chi \phi=\phi$, we get

$$
\begin{aligned}
\phi u & =\psi Q^{i D}\left(\chi P_{A} f\right)-\psi Q^{i D} P_{1} \chi \phi u \\
& =\psi Q^{i D}\left(\chi P_{A} f\right)+\left(-\psi Q^{i D} P_{1} \chi\right)\left(\psi Q^{i D}\left(\chi P_{A} f\right)-\psi Q^{i D} P_{1} \chi \phi u\right) \\
& =\sum_{j=0}^{n}\left(-\psi Q^{i D} P_{1} \chi\right)^{j}\left(\psi Q^{i D}\left(\chi P_{A} f\right)+\left(-\psi Q^{i D} P_{1} \chi \phi u\right)^{n+1} .\right.
\end{aligned}
$$

The operator norm has $\left\|\psi Q^{i D} P_{1} \chi\right\|_{L^{2}((0, \varepsilon), H)}<1 / 2$ for $\varepsilon$ sufficiently small, and $V=\sum_{j \geq 0}\left(-\psi Q^{i D} P_{1} \chi\right)^{j}$ is a bounded operator with $\phi u=V \psi Q^{i D} P_{A} \phi u$, i.e., $V \psi Q^{i D} P_{A}=I d$.

Theorem 3.5. (1) $P_{A}^{i D}$ is a closed operator, and

(2) $P_{A}^{i D}: \mathcal{D}^{i D}\left(P_{A}\right) \rightarrow L^{2}\left(\Omega^{1} \oplus \Omega^{0}\left(Y,\left.\operatorname{ad} P\right|_{Y}\right)\right)$ is a Fredholm operator.

Proof. (1) If $u_{n} \in \mathcal{D}^{i D}\left(P_{A}\right)$ with $u_{n} \rightarrow u$ and $P_{A}^{i D} u_{n} \rightarrow v$ in $L^{2}\left(\Omega^{1} \oplus \Omega^{0}(Y\right.$, $\left.\left.\operatorname{ad} P\right|_{Y}\right)$ ), then $u \in \mathcal{D}\left(P_{A, \max }\right)$ and $v=P_{A} u$ in $L^{2}\left(\Omega^{1} \oplus \Omega^{0}\left(Y,\left.\operatorname{ad} P\right|_{Y}\right)\right)$. For $\varepsilon$ in Lemma 3.4, $\phi \in C_{0}^{\infty}(-\varepsilon, \varepsilon)$ with $\phi=1$ near 0 , we get

$$
\begin{aligned}
\phi u_{n} & =V \psi Q^{i D}\left(P_{A} \phi u_{n}\right) \\
& =V \psi Q^{i D}\left(\phi^{\prime} u_{n}+\phi P_{A, \max } u_{n}\right) \\
& \rightarrow V \psi Q^{i D}\left(\phi^{\prime} u+\phi v\right) \\
& =\phi u,
\end{aligned}
$$

and $\|u(t)\|_{H}=o(1)$ as $t \rightarrow 0$. Thus $P_{A}^{i D}$ is a closed operator.

(2) By (1), $\mathcal{D}^{i D}\left(P_{A}\right)$ is a Hilbert space under the graph norm. It suffices to show that $P_{A}^{i D}$ has finite dimensional kernel and cokernel for the Fredholm property. This is amount to constructing right and left parametrices for $P_{A}^{i D}$.

Let $\phi, \tilde{\phi} \in C_{0}^{\infty}(-\varepsilon, \varepsilon)$ be such that $\phi=1$ near 0 and $\tilde{\phi}=1$ near $\operatorname{supp} \phi$, and $\psi, \tilde{\psi} \in C_{0}^{\infty}(M)$ such that $\phi+\psi=1$ and $\tilde{\psi}=1$ in a neighborhood of $\operatorname{supp} \psi$. Let $P_{i}: L^{2}\left(\Omega^{1} \oplus \Omega^{0}\left(Y,\left.\operatorname{ad} P\right|_{Y}\right)\right) \rightarrow L_{1, l o c}^{2}\left(\Omega^{1} \oplus \Omega^{0}\left(Y,\left.\operatorname{ad} P\right|_{Y}\right)\right)$ be an interior parametrix for $P_{A}$ (see $\left.[5,6]\right)$ with

$$
P_{A} \tilde{\psi} P_{i} \psi=\psi+R_{i} ; \quad \tilde{\psi} P_{i} \psi P_{A}=\psi+L_{i},
$$

where $R_{i}$ and $L_{i}$ are compact in $L^{2}\left(\Omega^{1} \oplus \Omega^{0}\left(Y,\left.\operatorname{ad} P\right|_{Y}\right)\right)$. Define $Q_{A}=\tilde{\phi} Q_{A}^{i D} \phi+$ $\tilde{\psi} P_{i} \psi$. By Lemma $3.2, Q_{A}^{i D}$ maps into $\mathcal{D}^{i D}\left(P_{A}\right)$ and

$$
\begin{aligned}
P_{A}^{i D} Q_{A} & =P_{A}^{i D}\left(\tilde{\phi} Q_{A}^{i D} \phi+\tilde{\psi} P_{i} \psi\right) \\
& =I d+\tilde{\phi} Q_{A}^{i D} \phi+\tilde{\phi} P_{1} Q_{A}^{i D} \phi+R_{i},
\end{aligned}
$$


where $\tilde{\phi}^{\prime}$ stands for $\left(\frac{\partial}{\partial t}+t^{-1} P_{0}\right) \tilde{\phi}$. If the supp $\phi$ is sufficiently small, then $\left\|\tilde{\phi}^{\prime} Q_{A}^{i D} \phi+\tilde{\phi} P_{1} Q_{A}^{i D} \phi\right\| \leq 1 / 4$ and $P_{A}^{i D} Q_{A}=I d+R+R_{i}$ with $R_{i}$ compact operator and $\|R\| \leq 1 / 4$. This implies that

$$
P_{A}^{i D} Q_{A}(I d+R)^{-1}=I d+R_{i}(I d+R)^{-1}
$$

and $P_{A}^{i D}$ has finite-dimensional cokernel.

On the other hand, by Lemma 3.2, we have, similarly,

$$
\begin{aligned}
Q_{A} P_{A}^{i D} & =\left(\tilde{\phi} Q_{A}^{i D} \phi+\tilde{\psi} P_{i} \psi\right) P_{A}^{i D} \\
& =I d+\left(\tilde{\phi} Q_{A}^{i D} P_{1} \phi-\tilde{\phi} Q_{A}^{i D} \phi^{\prime}\right)+L_{i},
\end{aligned}
$$

for sufficiently small $\varepsilon$,

$$
\|L\|=\left\|\left(\tilde{\phi} Q_{A}^{i D} P_{1} \phi-\tilde{\phi} Q_{A}^{i D} \phi^{\prime}\right)\right\| \leq 1 / 4,
$$

and $L_{i}$ is a compact operator. Hence

$$
(I d+L)^{-1} Q_{A} P_{A}^{i D}=I d+(I d+L)^{-1} L_{i},
$$

and $P_{A}^{i D}$ has finite-dimensional kernel. Thus the desired result follows.

The proof of Theorem 3.5 is standard in $[1,11]$. The result can also follow from the general theory in [11] for the differential operators of Fuchs type.

Corollary 3.6. Let $P(t) \in \mathcal{L}\left(H_{s}, H\right)$ be a smooth function of $t \in(0,1]$. Then for $\chi \in C_{0}^{\infty}(-\varepsilon, \varepsilon)$ with $\varepsilon$ sufficiently small and $\tilde{P}_{A}=P_{A}^{i D}+\chi t P(t)$ is a Fredholm operator on $\mathcal{D}^{i D}\left(P_{A}\right)$ with $\operatorname{Ind}\left(P_{A}^{i D}\right)=\operatorname{Ind}\left(\tilde{P}_{A}\right)$.

Proof. Note that $\chi t P(t)=P(t)\left(\left|P_{0}\right|+1\right)^{-1} \chi t\left(\left|P_{0}\right|+1\right)$ is bounded from $\mathcal{D}^{i D}\left(P_{A}\right)$ to $L^{2}\left(\Omega^{1} \oplus \Omega^{0}\left(Y,\left.\operatorname{ad} P\right|_{Y}\right)\right)$. The family $P_{A}^{i D}(s)=P_{A}^{i D}+s \chi t P(t)$ is a continuous function of $s \in[0,1]$ with values in $\mathcal{L}\left(\mathcal{D}^{i D}\left(P_{A}\right), L^{2}\left(\Omega^{1} \oplus \Omega^{0}\left(Y,\left.\operatorname{ad} P\right|_{Y}\right)\right)\right)$. By Theorem 3.5, with $\tilde{\psi}$ such that $\chi \tilde{\psi}=0, P_{A}^{i D}(s)$ is a Fredholm operator for each $s \in[0,1]$ and the index is constant.

Therefore the index of $P_{A}^{i D}$ can be conveniently computed by the index of the operator $\frac{\partial}{\partial t}+t^{-1} P_{0}$ without $P_{1}$ term. We will apply Corollary 3.6 in section 5 for the index calculation.

\section{Closed Extensions for $P_{A}$}

For the operator $P_{A}=\frac{\partial}{\partial t}+t^{-1} P_{0}+P_{1}$, we have the formal adjoint

$$
P_{A}^{*}=-\frac{\partial}{\partial t}+t^{-1} P_{0}^{*}+P_{1}^{*}
$$

where $P_{0}$ and $P_{1}$ are self-adjoint due to the self-adjointness of $P_{0}+t P_{1}$.

Lemma 4.1. If $u \in \mathcal{D}^{i D}\left(P_{A}\right)$ with $u \equiv 0$ for $t \geq 1$, then there exists a operator $Q_{A, \max }=\oplus_{s<1 / 2} P_{1, s} \oplus \oplus_{s \geq 1 / 2} P_{0, s}$ such that

$$
Q_{A, \max } P_{A} u=u+\left(Q_{A, \max } P_{1}\right) u,
$$

with $Q_{A, \max } P_{1}$ is bounded in $L^{2}((0,1), H)$ and $s \in \operatorname{Spec} P_{0}$. 
Proof. Similar to Lemma 3.2, we take an orthonormal basis of eigensections $\left\{e_{s}\right\}_{s \in \operatorname{Spec} P_{0}}$. Let $u, P_{A} u \in L^{2}$. Define

$$
h(t)=u^{\prime}(t)+t^{-1} P_{0} u(t)=P_{A} u(t)-P_{1}(u(t)) .
$$

By the uniform estimates, one gets

$$
\begin{aligned}
\left\|P_{1} e_{s}\right\|_{H} & =\left\|P_{1}\left(\left|P_{0}\right|+1\right)^{-1}\left(\left|P_{0}\right|+1\right) e_{s}\right\|_{H} \\
& \leq C\left\|\left(\left|P_{0}\right|+1\right) e_{s}\right\|_{H} \\
& \leq C(|s|+1) .
\end{aligned}
$$

Hence for each $s \in \operatorname{Spec} P_{0}, h_{s}(t)=\left\langle h(t), e_{s}\right\rangle_{H}$ is given by

$$
h_{s}(t)=\left\langle P_{A} u, e_{s}\right\rangle-\left\langle u, P_{1}^{*} e_{s}\right\rangle \in L^{2}
$$

since both $u$ and $P_{A} u$ are in $L^{2}$. By definition $u(t) \equiv 0$ for $t \geq 1, u_{s}(t) \equiv 0$ for $t \geq 1$, and for $s<1 / 2$,

$$
P_{1, s} h_{s}=P_{1, s}\left(u_{s}^{\prime}+t^{-1} s u_{s}\right)=u_{s} \in L^{2} .
$$

For $s \geq 1 / 2$, using integration by parts we have

$$
t^{-s} \int_{0}^{1} x^{s} h_{s}(x) d x=P_{0, s} h_{s}(t)-P_{1, s} h_{s}(t) \in L^{2} .
$$

By the same argument in Lemma 3.3, $\int_{0}^{1} x^{s} h_{s}(x) d x=0$. Hence $P_{0, s} h_{s}=$ $P_{1, s} h_{s}=u_{s}$ for $s \geq 1 / 2$. For all $s \in \operatorname{Spec} P_{0}, Q_{A, \max } h=u$ gives the desired equality

$$
Q_{A, \max } P_{A} u=u+\left(Q_{A, \max } P_{1}\right) u .
$$

Theorem 4.2. (1) $P_{A, \max }$ and $P_{A, \min }$ are Fredholm operators;

(2) The extensions of $P_{A, \min }$ are all Fredholm operators and correspond to the subspaces of the finite-dimensional space $\mathcal{D}^{i D}\left(P_{A}\right) / \mathcal{D}^{i D}\left(P_{A}^{*}\right)$.

Proof. Choose $\phi, \tilde{p}, \psi, \tilde{\psi}$ as in Theorem 3.5, and define the parametrix $Q=$ $\tilde{\phi} Q_{A, \max } \phi+\tilde{\psi} P_{i} \psi$. Then, by Lemma 4.1 , we have

$$
\begin{aligned}
Q P_{A, \max } u & =\left(\tilde{\phi} Q_{A, \max } \phi+\tilde{\psi} P_{i} \psi\right) P_{A, \max } u \\
& =u+\left(\tilde{\phi} Q_{A, \max } P_{1} \phi-\tilde{\phi} Q_{A, \max } \phi^{\prime}\right) u+L_{i} u .
\end{aligned}
$$

By the same estimates in Theorem 3.5, the operator $\left(\tilde{\phi} Q_{A, \max } P_{1} \phi-\tilde{\phi} Q_{A, \max } \phi^{\prime}\right)$ has norm $\leq 1 / 4$ and $L_{i}$ is compact. Therefore $Q P_{A, \max }$ is Fredholm with finite nullity. So the operator $P_{A, \max }$ has finite nullity. Since it is an extension of the Fredholm operator $P_{A}^{i D}$, it also has closed range with finite codimension. Thus $P_{A, \max }$ is Fredholm.

The same argument applies to $P_{A, \max }^{*}$ and its adjoint $P_{A, \min }$ is Fredholm too. Note that $\mathcal{D}\left(P_{A, \min }\right) \subset \mathcal{D}\left(P_{A, \max }\right)$ with finite codimension since both operators $P_{A, \max }$ and $P_{A, \min }$ are Fredholm. Thus

$$
\operatorname{Ind}\left(P_{A, \max }\right)=\operatorname{Ind}\left(P_{A, \min }\right)+\operatorname{dim} \mathcal{D}^{i D}\left(P_{A, \max }\right) / \mathcal{D}^{i D}\left(P_{A, \min }\right),
$$


where $\mathcal{D}^{i D}\left(P_{A, \min }\right)=\mathcal{D}^{i D}\left(P_{A}^{*}\right)$ and $\mathcal{D}^{i D}\left(P_{A, \max }\right)=\mathcal{D}^{i D}\left(P_{A}\right)$.

The finite-dimensional space $\mathcal{D}^{i D}\left(P_{A, \max }\right) / \mathcal{D}^{i D}\left(P_{A, \min }\right)$ is isomorphic to $\oplus_{|s|<1 / 2} \operatorname{ker}\left(P_{0}-s\right)$ by results in $[1,11]$. Closed extensions of $P_{A, \min }$ are determined by the asymptotic behavior of the elements in their domains.

Lemma 4.3. For $s \in \operatorname{Spec} P_{0}$ with $|s|<1 / 2$, there are $C^{0}$-linear functionals $c_{s}$ on $\mathcal{D}\left(P_{A, \max }\right)$ such that for $t \in(0,1), 0<\varepsilon<1$ and $u \in \mathcal{D}\left(P_{A, \max }\right)$,

$$
\left\|u(t)-\sum_{|s|<1 / 2} c_{s}(u) t^{-s} e_{s}\right\|_{H} \leq \varepsilon \sqrt{t|\ln t|}+C_{\varepsilon, u} \sqrt{t}
$$

The same estimate holds for $P_{A}^{*}$, mutatis mutandis.

Proof. By the same argument of applying Lemma 3.4 as in Theorem 3.5, Lemma 3.2 implies that $u$ is an element of $\mathcal{D}\left(P_{A, \max }\right)$. Thus $\left\|\left(\left|P_{0}\right|+1\right) u(t)\right\|_{H} \in$ $L^{2}((0,1))$ and

$$
\int_{0}^{1}\left\|\left(\left|P_{0}\right|+1\right) u(t)\right\|_{H}^{2} d t \leq C\left(\left\|P_{A} u\right\|^{2}+\|u\|^{2}\right) .
$$

For $s>-1 / 2, h=u^{\prime}+t^{-1} P_{0} u$, there is a $c_{s}(u)$ with

$$
u_{s}(t)=t^{-s}\left[c_{s}(u)+\int_{0}^{t} x^{s} h_{s}(x) d x\right]
$$

from integration by parts. Since $\int_{0}^{t}\left(\frac{x}{t}\right)^{s} h_{s}(x) d x \in L^{2}$ by Lemma 3.2 , for $s \geq$ $1 / 2, c_{s}(u)=0$. For $|s|<1 / 2$ and let $t=1$,

$$
c_{s}(u)=u_{s}(1)-\int_{0}^{1} x^{s} h_{s}(x) d x
$$

and $u_{s}(t)=t^{-s} u_{s}(1)+P_{1, s} h_{s}(t)$ for $s<1 / 2$. By interior regularity, $u^{\prime} \in$ $L^{2}((1 / 2,1), H)$ and

$$
\begin{gathered}
\left|u_{s}(1)\right|^{2} \leq t^{s}\left|u_{s}(t)\right|^{2}+\left|P_{1, s} h_{s}(t)\right|^{2} \\
\|u(1)\|_{H}^{2} \leq C \int_{1 / 2}^{1}\left(\|u(t)\|_{H}^{2}+\left\|u^{\prime}(t)\right\|_{H}^{2}\right) d t .
\end{gathered}
$$

Hence $c_{s}(u)$ is continuous on $\mathcal{D}^{i D}\left(P_{A}\right)$, and

$$
\sum_{s \leq-1 / 2} t^{-2 s}\left|u_{s}(1)\right|^{2} \leq C_{u} t
$$

Combining Lemma 3.1, for $0<\delta<1$,

$$
\sum_{s \leq-1 / 2}\left|P_{1, s} h_{s}(t)\right|^{2} \leq t\left[\|h\|_{H}^{2}+2|\ln \delta|\left\|h_{-1 / 2}\right\|_{H}^{2}+2|\ln t| \int_{0}^{\delta}\left|h_{-1 / 2}\right|^{2} d x\right] .
$$

By Lemma 3.1 (1)

$$
\sum_{s>-1 / 2}\left|u_{s}(t)-c_{s}(u) t^{-s}\right|^{2}=\sum_{s>-1 / 2}\left|P_{0, s} h_{s}(t)\right|^{2} \leq C t\|h\|_{H}^{2} .
$$


Thus the result follows.

With Lemma 4.3 , we can define various extensions of $P_{A, \min }$ and compute their indexes in terms of the extension determined by the subspaces of $\oplus_{|s|<1 / 2} \operatorname{ker}\left(P_{0}-s\right)$. Let $P_{A, W}$ be an extension of $P_{A, \min }$ by restricting $P_{A, \max }$ to the domain $\mathcal{D}\left(P_{A, W}\right)=\left\{u \in \mathcal{D}\left(P_{A, \max }\right) \mid \sum_{|s|<1 / 2} c_{s}(u) e_{s} \in W\right\}$, where $W$ is a subspace of $\oplus_{|s|<1 / 2} \operatorname{ker}\left(P_{0}-s\right)$. So $P_{A, W}$ is automatically closed since the functionals $c_{s}$ are continuous on the domain $\mathcal{D}\left(P_{A, \max }\right)$. Our main result of this section is the following theorem.

Theorem 4.4. The operators $P_{A, W}$ give all closed extensions of $P_{A, \min }$ for all subspace $W \subset \oplus_{|s|<1 / 2} \operatorname{ker}\left(P_{0}-s\right) . \quad P_{A, W}^{*}=P_{A, W \perp}$, where $W^{\perp}$ is the complement subspace of $W$ in $\oplus_{|s|<1 / 2} \operatorname{ker}\left(P_{0}-s\right)$. Furthermore,

$$
\text { Ind }\left(P_{A, W}\right)=\operatorname{Ind}\left(P_{A, \min }\right)+\operatorname{dim} W .
$$

Proof. For $u \in \mathcal{D}\left(P_{A, \text { max }}\right)$ and $v \in \mathcal{D}\left(P_{A, \text { max }}^{*}\right)$,

$$
\left\langle P_{A} u, v\right\rangle=\left\langle u, P_{A}^{*} v\right\rangle-\sum_{|s|<1 / 2} c_{s}(u) c_{-s}^{*}(v),
$$

where $c_{s}^{*}$ are functionals for $P_{A, \max }^{*}$ on the domain $\mathcal{D}\left(P_{A, \max }^{*}\right)$. By Lemma 4.3, with $\phi \in C_{0}^{\infty}(-1,1)$ and $\phi \equiv 0$ near $t=0$,

$$
\begin{aligned}
& u(t)=\phi(t) \sum_{|s|<1 / 2} c_{s}(u) t^{-s} e_{s}+\tilde{u}(t), \\
& v(t)=\phi(t) \sum_{|s|<1 / 2} c_{-s}^{*}(v) t^{s} e_{s}+\tilde{v}(t),
\end{aligned}
$$

with $\|\tilde{u}(t)\|+\|\tilde{v}(t)\| \leq C \sqrt{t|\ln t|}$ as $t \rightarrow 0$. Thus we obtain

$$
\begin{aligned}
\left\langle P_{A} u, v\right\rangle & =\lim _{\varepsilon \rightarrow 0} \int_{\varepsilon}^{1}\left\langle P_{A} u, \phi v\right\rangle_{H}+\left\langle u, P_{A}^{*}(1-\phi) v\right\rangle \\
& =\lim _{\varepsilon \rightarrow 0}-\langle u(\varepsilon), v(\varepsilon)\rangle_{H}+\left\langle u, P_{A}^{*} v\right\rangle \\
& =\left\langle u, P_{A}^{*} v\right\rangle-\sum_{|s|<1 / 2} c_{s}(u) c_{-s}^{*}(v) .
\end{aligned}
$$

For $|s|<1 / 2$ with any constant $c_{s}$, set $u(t)=\phi \sum_{|s|<1 / 2} c_{s} t^{-s} e_{s}$. Then one can compute the term

$$
P_{A} u(t)=\phi^{\prime}(t) \sum_{|s|<1 / 2} c_{s} t^{-s} e_{s}+\phi \sum_{|s|<1 / 2} c_{s} t^{-s}\left[P_{1}\left(\left|P_{0}\right|+1\right)^{-1}\right](|s|+1) e_{s} \in L^{2} .
$$

Note that $P_{A, \max }^{*}=\left(P_{A, \min }\right)^{*}$ and $\left(P_{A, \max }^{*}\right)^{*}=P_{A, \min } . c_{s}(u)=0$ for all $s$ if and only if $u \in \mathcal{D}\left(P_{A, \min }\right)$. Thus the equation (8) implies $u \in \mathcal{D}\left(\left(P_{A, \max }^{*}\right)^{*}\right)$, the converse follows from the continuous functional $c_{s}$.

For any extension $P$ of $P_{A, \min }$, define $W=\left\{\sum_{|s|<1 / 2} c_{s}(u) e_{s} \mid u \in \mathcal{D}(P)\right\}$. Then it is clear that $P \subset P_{A, W}$. For $v \in \mathcal{D}\left(P_{A, W}\right)$, there is an element $u \in \mathcal{D}(P)$ such that $c_{s}(v-u)=0$ for all $s$ by definition. So $u-v \in \mathcal{D}\left(P_{A, \min }\right) \subset \mathcal{D}(P)$ by 
the above necessary and sufficient condition, therefore $P=P_{A, W}$. The index formula for $P_{A, W}$ is clear from Theorem 4.2 and $\mathcal{D}\left(P_{A, W}\right) / \mathcal{D}\left(P_{A, \min }\right) \cong W$.

Note that for $u=(\alpha, \phi), P_{0} u=s u$ is equivalent to the system of equations:

$$
\begin{aligned}
{ }^{*} d_{a_{0}} \alpha+d_{a_{0}} \phi & =(s+1 / 2) \alpha \\
d_{a_{0}}^{*} \alpha & =(s-3 / 2) \phi
\end{aligned} .
$$

Thus, by applying $d_{a_{0}}^{* Y}$ to the first equation, one gets

$$
\Delta^{0} \phi=d_{a_{0}}^{*_{Y}} d_{a_{0}} \phi=(s+1 / 2) d_{a_{0}}^{*_{Y}} \alpha=(s+1 / 2)(s-3 / 2) \phi .
$$

Let $H_{\lambda(s)}^{0}=\left\{\phi \in \Omega^{0}\left(Y,\left.\operatorname{ad} P\right|_{Y}\right) \mid \Delta^{0} \phi=\lambda(s) \phi\right\}$. Hence for the nonnegative operator $\Delta^{0}$ the eigenvalues $\lambda(s)=(s+1 / 2)(s-3 / 2) \geq 0$. So $s \in(-\infty,-1 / 2] \cup$ $[3 / 2, \infty)$ if and only if $\lambda(s) \geq 0$. For $-1 / 2<s<3 / 2, H_{\lambda(s)}^{0}=\{0\}$.

Corollary 4.5. Let $H_{\lambda, c c}^{1}=\left\{\alpha \in \Omega^{1}\left(Y,\left.\operatorname{ad} P\right|_{Y}\right) \mid d_{a_{0}}^{*_{Y}} \alpha=0, \Delta^{1}=\lambda \alpha\right\}$. Then we have

$$
\oplus_{|s|<1 / 2} \operatorname{ker}\left(P_{0}-s\right) \cong \oplus_{|s|<1 / 2} H_{(s+1 / 2)^{2}, c c}^{1} .
$$

In particular, if $\oplus_{|s|<1 / 2} H_{(s+1 / 2)^{2}, c c}^{1}=\{0\}$, then there is a unique closed extension $\bar{P}_{A}$ of $P_{A}$, where $\Delta^{i}$ is the Laplacian operator on $\Omega^{i}\left(Y,\left.\operatorname{ad} P\right|_{Y}\right)$ with the twisted connection $a_{0}(i=0,1)$.

Proof. It suffices to see $\oplus_{|s|<1 / 2} \operatorname{ker}\left(P_{0}-s\right)=\{0\}$. For $|s|<1 / 2$, one must have $\phi \equiv 0$ since $\lambda(s)<0$ from the above discussion. Thus the equations reduce to

$$
*_{Y} d_{a_{0}} \alpha=(s+1 / 2) \alpha, \quad d_{a_{0}}^{*_{Y}} \alpha=0 .
$$

Thus the space $\oplus_{|s|<1 / 2} \operatorname{ker}\left(P_{0}-s\right)$ can be reduced to $\{(\alpha, \phi): \phi=0, \alpha \in$ $\left.H_{(s+1 / 2)^{2}, c c}^{1}\right\}$. The results follow.

\section{The index calculation for $P_{A}$}

By Corollary 3.6, the index of $P_{A}$ with respect to a closed extension in $\S 4$ can be reduced to the index of $\frac{\partial}{\partial t}+t^{-1} P_{0}$ with respect to the closed extension. By Theorem 4.4, we can just calculate the index of $P_{A}^{i D}$ (the restriction of $P_{A, \max }$ to the ideal boundary Dirichlet domain $\mathcal{D}^{i D}\left(P_{A}\right)$ ) with $P_{1} \equiv 0$.

Note that $P_{A}^{i D}$ is closed and $\Delta_{A}^{+}=\left(P_{A}^{i D}\right)^{*} P_{A}^{i D}, \Delta_{A}^{-}=P_{A}^{i D}\left(P_{A}^{i D}\right)^{*}$ are nonnegative, self-adjoint. By a straightforward calculation, one gets

$$
\begin{aligned}
& \Delta_{A}^{+}=-\frac{\partial^{2}}{\partial t^{2}}+t^{-2}\left(P_{0}^{2}+P_{0}\right), \\
& \Delta_{A}^{-}=-\frac{\partial^{2}}{\partial t^{2}}+t^{-2}\left(P_{0}^{2}-P_{0}\right) .
\end{aligned}
$$

By the completing square, we have $P_{0}^{2} \pm P_{0} \geq 1 / 4$. Any nonzero eigenvalue of $\Delta_{A}^{+}$and $\Delta_{A}^{-}$coincide with same multiplicity, i.e., $u \mapsto P_{A}^{i D} u$ and $v \mapsto\left(P_{A}^{i D}\right)^{*} v$ are injective between the corresponding eigenspaces. The idea is to show that $\left(\Delta_{A}^{ \pm}+\lambda\right)^{-m}$ are trace class and then

$$
\operatorname{tr}\left(\Delta_{A}^{+}+\lambda\right)^{-m}-\operatorname{tr}\left(\Delta_{A}^{-}+\lambda\right)^{-m}=\lambda^{-m} \operatorname{Ind}\left(P_{A}^{i D}\right)
$$


for appropriate $m$, since all terms approach to zero as $\lambda \rightarrow 0$ except the term $\lambda^{-m}$.

Let $\Delta_{A}^{+}$be the operator in $L^{2}\left(R_{+}, H\right)$ with the boundary condition $u(t)=$ $o(1)$ and $P_{A}^{i D} u=\frac{\partial u}{\partial t}+t^{-1} P_{0} u=O(1)$. Similarly for $\Delta_{A}^{-}$with $u(t)=o(1)$ and $\left(P_{A}^{i D}\right)^{*} u=-\frac{\partial u}{\partial t}+t^{-1} P_{0} u=o(1)$. By the standard result in [12], the resolvent for $\Delta_{A}^{ \pm}$is obtained by

$$
\left(\Delta_{A}^{ \pm}+\lambda\right)^{-1}=\oplus_{s \in \operatorname{Spec} P_{0}}\left(\Delta_{s}^{ \pm}+\lambda\right)^{-1} \otimes \pi_{s},
$$

where $\Delta_{s}^{ \pm}=-\frac{\partial^{2}}{\partial t^{2}}+t^{-2}\left(s^{2} \pm s\right)$ and $\pi_{s}$ is the projection on the $s$-eigenspace of $P_{0}$. Note that

$$
\begin{aligned}
& P_{0}^{2}+P_{0}=\left(\begin{array}{cc}
\Delta^{1} & 0 \\
0 & \Delta^{0}
\end{array}\right)+2\left(\begin{array}{cc}
0 & d_{a_{0}} \\
d_{a_{0}}^{* Y} & 0
\end{array}\right)+\left(\begin{array}{cc}
-\frac{1}{4} & 0 \\
0 & \frac{15}{4}
\end{array}\right) \\
& P_{0}^{2}-P_{0}=\left(\begin{array}{cc}
\Delta^{1} & 0 \\
0 & \Delta^{0}
\end{array}\right)-2\left(\begin{array}{cc}
*_{Y} d_{a_{0}} & 0 \\
0 & 0
\end{array}\right)+\left(\begin{array}{cc}
\frac{3}{4} & 0 \\
0 & \frac{3}{4}
\end{array}\right),
\end{aligned}
$$

where $\Delta^{i}$ is the Laplacian operator on $\Omega^{i}\left(Y,\left.\operatorname{ad} P\right|_{Y}\right)$ with the twisted connection $a_{0}$.

Let us first compute the resolvent kernel of $\Delta_{s}^{+}+z^{2}$. Let $u_{1}$ and $u_{2}$ be the independent solutions, $0<t<\infty$,

$$
\begin{cases}\left(-\frac{\partial^{2}}{\partial t^{2}}+t^{-2}\left(s^{2}+s\right)+z^{2}\right) u & =0 \\ u^{\prime}(t)+t^{-1} P_{0} u(t)=O(1) & u(t)=o(1)\end{cases}
$$

By Theorem 16 of Chapter XIII of [7] in page 1329 (or $\S 8.4$ of [12]), the resolvent is given by

$$
\left(\Delta_{s}^{+}+z^{2}\right)^{-1} f(t)=R(z, s) f=\int_{0}^{\infty} f(x) K(t, x ; z) d x
$$

for $f \in L_{2}(0, \infty)$, and the kernel is given by

$$
K(t, x ; z)= \begin{cases}\frac{u_{1}(t, z) u_{2}(x, z)}{W\left(u_{1}, u_{2}\right)} & x<t \\ \frac{u_{1}(x, z) u_{2}(t, z)}{W\left(u_{1}, u_{2}\right)} & x>t\end{cases}
$$

where $W\left(u_{1}, u_{2}\right)=u_{1}^{\prime} u_{2}-u_{1} u_{2}^{\prime}$ is the Wronskian of $u_{1}$ and $u_{2}$. By substituting $u_{i}=t^{1 / 2} v_{i}$, so $v_{1}, v_{2}$ are fundamental solutions of

$$
-t^{2} v^{\prime \prime}-t v^{\prime}+\left((s+1 / 2)^{2}+(t z)^{2}\right) v=0 .
$$

Hence the general solution can be expressed $u=c_{1} t^{1 / 2} v_{1}+c_{2} t^{1 / 2} v_{2}$. Theorem 16 of [7] shows that there is exactly one solution $\psi(t, z)=t^{1 / 2} v_{2}(t z)$ satisfying the condition at $t=\infty$. Unless $-1 / 2<s<0, \phi(t, z)=t^{1 / 2} v_{1}(t z)$ is the unique solution satisfying the condition as $t \rightarrow 0$. Since $W(\phi, \psi)=t W\left(v_{1}, v_{2}\right)$ 
and $v_{1}, v_{2}$ are solutions of $(9)$, one gets

$$
\begin{aligned}
t v_{1}^{\prime} v_{2} & =\left(-t^{2} v_{1}^{\prime \prime}+\left((s+1 / 2)^{2}+(t z)^{2}\right) v_{1}\right) v_{2} \\
& \left.=-t^{2} v_{1}^{\prime \prime} v_{2}+v_{1}\left((s+1 / 2)^{2}+(t z)^{2}\right)\right) v_{2} \\
& =-t^{2} v_{1}^{\prime \prime} v_{2}+v_{1}\left(t^{2} v_{2}^{\prime \prime}+t v_{2}^{\prime}\right)
\end{aligned}
$$

Hence $v_{1}^{\prime} v_{2}-v_{1} v_{2}^{\prime}=-t\left(v_{1}^{\prime} v_{2}-v_{1} v_{2}^{\prime}\right)^{\prime}$ and $v_{1}^{\prime} v_{2}-v_{1} v_{2}^{\prime}=1 / t$. So $W(\phi, \psi)=1$ and the kernel is

$$
K(t, x ; z)=\left\{\begin{array}{l}
\frac{\phi(t, z) \psi(x, z)}{W(\phi, \psi)} \\
\frac{\phi(x, z) \psi(t, z)}{W(\phi, \psi)}
\end{array}= \begin{cases}(t x)^{1 / 2} v_{1}(t z) v_{2}(x z) & x<t \\
(t x)^{1 / 2} v_{1}(x z) v_{2}(t z) & x>t\end{cases}\right.
$$

The solutions $v_{1}$ and $v_{2}$ are depending on $s$ and $z$. Let $\nu_{ \pm}=|s \pm 1 / 2|$. If $\operatorname{Im} z^{2} \neq 0, t \leq x$, , the Kernel $K^{+}(t, x ; z)$ of $\left(\Delta_{s}^{+}+z^{2}\right)^{-1}$ can be expressed as

$$
\begin{cases}(t x)^{1 / 2} v_{1, \nu_{+}}(t z) v_{2, \nu_{+}}(x z) & \text { if } s \leq-1 / 2 \text { or } s \geq 0 \\ (t x)^{1 / 2} v_{1,-\nu_{+}}(t z) v_{2, \nu_{+}}(x z) & \text { if }-1 / 2<s<0\end{cases}
$$

Similarly, the kernel $K^{-}(t, x ; z)$ of $\left(\Delta_{s}^{-}+z^{2}\right)^{-1}$ is

$$
\begin{cases}(t x)^{1 / 2} v_{1, \nu_{-}}(t z) v_{2, \nu_{-}}(x z) & \text { if } s<0 \text { or } s \geq 1 / 2 \\ (t x)^{1 / 2} v_{1,-\nu_{-}}(t z) v_{2, \nu_{-}}(x z) & \text { if } 0 \leq s<1 / 2\end{cases}
$$

Let $P_{i}^{ \pm}$be the standard pseudo-differential parametrix for $\left(\Delta_{s}^{ \pm}+\lambda\right)^{-m}$ in the interior away from $t=0$. If $\phi, \psi \in C^{\infty}$ vanishing near $t=0$ and $\psi \equiv 1$ near $\operatorname{supp} \phi$, then

$$
\left(\Delta_{s}^{ \pm}+\lambda\right)^{m} \psi P_{i}^{ \pm} \phi=\phi-R_{i}^{ \pm},
$$

with $\left\|R_{i}\right\|_{\text {tr }}=O\left(\lambda^{-k}\right)$, where $k$ can be arbitrarily large. Choose $\chi \in C_{0}^{\infty}(-1$, $x_{0}$ ) with $x_{0}<\varepsilon$ and $\chi \equiv 1$ near $t=0$ and $\tilde{\chi} \in C_{0}^{\infty}(-1, \varepsilon)$ with $\tilde{\chi} \equiv 1$ for $0 \leq t \leq t_{0}$ with $t_{0}>x_{0}$. Let $\chi_{i}=1-\chi$ and $\tilde{\chi}_{i} \in C_{0}^{\infty}(X)$ be vanishing near $t=0$ with $\tilde{\chi}_{i} \equiv 1$ near $\operatorname{supp} \chi_{i}$. Define

$$
P^{ \pm}=\tilde{\chi}\left(P_{A}+\lambda\right)^{-m} \chi+\tilde{\chi}_{i} P_{i}^{ \pm} \chi_{i} .
$$

Then $\left(\Delta_{A}^{ \pm}+\lambda\right) P^{ \pm}=I d-R^{ \pm}$, where

$$
R^{ \pm}=R_{i}^{ \pm}+\sum_{j>0} c_{\alpha \beta \gamma, j k} \chi^{(j)} t^{\alpha} \frac{\partial^{\beta}}{\partial t^{\beta}} P_{0}^{\gamma}\left(P_{A}+\lambda\right)^{-k} \chi
$$

By Lemma 4.2 of [1], $\left\|R^{ \pm}\right\|_{\text {tr }} \leq C \lambda^{-k}$. Hence for large $\lambda,\left(\Delta_{A}^{ \pm}+\lambda\right)^{-m}=$ $P^{ \pm}\left(\sum_{j \geq 0}\left(R^{ \pm}\right)^{j}\right)$ with $\left\|P \sum_{j \geq 1}\left(R^{ \pm}\right)^{j}\right\|_{\text {tr }} \leq C \lambda^{-k}$. The asymptotic of $\operatorname{tr}\left(\Delta_{A}^{ \pm}+\right.$ $\lambda)^{-m}$ from $P^{ \pm}$is required for the calculation. Note that

$$
\left(\Delta_{A}^{ \pm}+\lambda\right)^{-m}=\frac{1}{(m-1) !}\left(-\frac{1}{2 z} \frac{\partial}{\partial z}\right)^{m-1}\left(\left(\Delta_{A}^{ \pm}+\lambda\right)^{-1} .\right.
$$


Substitute $\xi=t z$ and define formally,

$$
\begin{aligned}
\sigma_{+}(t, \xi)= & \frac{t^{2 m-1}}{(m-1) !}\left(-\frac{1}{\xi} \frac{\partial}{\partial \xi}\right)^{(m-1)}\left[\sum_{s \leq-1 / 2}+\sum_{s \geq 0} v_{1, \nu_{+}}(\xi) v_{2, \nu_{+}}(\xi)\right. \\
& \left.+\sum_{-1 / 2<s<0} v_{1,-\nu_{+}}(\xi) v_{2, \nu_{+}}(\xi)\right] \\
\sigma_{-}(t, \xi)= & \frac{t^{2 m-1}}{(m-1) !}\left(-\frac{1}{\xi} \frac{\partial}{\partial \xi}\right)^{(m-1)}\left[\sum_{s \geq 1 / 2}+\sum_{s<0} v_{1, \nu_{-}}(\xi) v_{2, \nu_{-}}(\xi)\right. \\
& \left.+\sum_{0 \leq s<1 / 2} v_{1,-\nu_{-}}(\xi) v_{2, \nu_{-}}(\xi)\right]
\end{aligned}
$$

following [1, 2]. Applying the same method in [1, 2], one obtains

(10) Ind $P_{A}^{i D}=\int_{X} \chi_{i} \omega\left(P_{A}\right)+\int_{0}^{\infty} \frac{\xi^{2 m-1}}{(2 m-1) !}\left[\sigma_{+}^{(2 m-1)}(0, \xi)-\sigma_{-}^{(2 m-1)}(0, \xi)\right] d \xi$,

where $\omega\left(P_{A}\right)$ is the local index form of the self-duality operator $P_{A}$ on $X$ and the second integral is given by

$$
-\frac{1}{2}\left(\eta_{P_{0}}+\operatorname{dim} \operatorname{ker} P_{0}\right)+\sum_{k \geq 1} \alpha_{k} \operatorname{Res}_{1} \eta_{P_{0}}(2 k)
$$

where $\alpha_{k}=\frac{2^{-2 k}}{2 k(2 k+1)}$ and the eta function of $P_{0}$ is defined by

$$
\eta_{P_{0}}(z)=\sum_{s \in \operatorname{Spec} P_{0} \backslash\{0\}}|s|^{-z} \text { sgns }
$$

in which $\eta_{P_{0}}(z)$ is meromorphic in the whole plane with possible simple poles but holomorphic at $z=0$, and the $\eta$-invariant of $P_{0}, \eta_{P_{0}}=\eta_{P_{0}}(0)$, is welldefined.

With $P_{1} \equiv 0$ for $t \leq \varepsilon$, the index formula of $P_{A}$ is given by

$$
\text { Ind } \begin{aligned}
P_{A}^{i D}= & -\frac{1}{2}\left(\eta_{P_{0}}+\operatorname{dim} \operatorname{ker} P_{0}\right)+\sum_{k \geq 1} \alpha_{k} \operatorname{Res}_{1} \eta_{P_{0}}(2 k) \\
& +\int_{X \backslash\{t \leq E\}} \frac{1}{8 \pi^{2}} \operatorname{Tr}\left(F_{A} \wedge F_{A}\right)
\end{aligned}
$$

following from (10) and (11). Define $\int_{X} \tilde{c}_{2}(\operatorname{ad} P)$ to be the limit

$$
\lim _{\varepsilon \rightarrow 0} \int_{t>\varepsilon} c_{2}(\operatorname{ad} P)=\lim _{\varepsilon \rightarrow 0} \int_{t>\varepsilon} \frac{1}{8 \pi^{2}} \operatorname{Tr}\left(F_{A} \wedge F_{A}\right) .
$$

Theorem 5.1. With the conic metric $d t^{2}+t^{2} g_{Y}$ at a point on $X$, the selfduality operator $P_{A}^{i D}$ is Fredholm with index

$$
\int_{X} \tilde{c}_{2}(\operatorname{ad} P)-\frac{1}{2}\left(\eta_{P_{0}}+m_{1}\right)+\sum_{k \geq 1} \frac{2^{-2 k}}{2 k(2 k+1)} \operatorname{Res}_{1} \eta_{P_{0}}(2 k),
$$


where $\eta_{P_{0}}(z)$ is the eta function of the operator $P_{0}$ and $m_{1}=\operatorname{dim} H_{1 / 4, c c}^{1}$ is the multiplicity of eigenvalue $1 / 4$ of the Laplacian operator $\Delta^{1}$ on the coclosed $\Omega^{1}\left(Y,\left.\operatorname{ad} P\right|_{Y}\right)$ with the twisted connection $a_{0}$.

Proof. ker $P_{0}$ can be identified with $H_{1 / 4, c c}^{1}$ by Corollary 4.5. The result follows from the above discussion and the method in $[1,2]$ as well as (12).

Corollary 5.2. The closed extension $P_{A, W}$ of $P_{A}$ is Fredholm with index

$$
\begin{aligned}
& \int_{X} \tilde{c}_{2}(\operatorname{ad} P)-\frac{1}{2}\left(\eta_{P_{0}}+m_{1}\right)+\sum_{k \geq 1} \frac{2^{-2 k}}{2 k(2 k+1)} \operatorname{Res}_{1} \eta_{P_{0}}(2 k) \\
& +\operatorname{dim} W-\sum_{-1 / 2<s<0} \operatorname{dim} \operatorname{ker}\left(P_{0}-s\right) .
\end{aligned}
$$

Proof. Let $W \subset \oplus_{|s|<1 / 2} \operatorname{ker}\left(P_{0}-s\right)$ be a subspace with

$$
W^{-}=\oplus_{-1 / 2<s<0} \operatorname{ker}\left(P_{0}-s\right), \quad W^{+}=\oplus_{1 / 2>s \geq 0} \operatorname{ker}\left(P_{0}-s\right) .
$$

Note that $\mathcal{D}\left(P_{A}^{i D}\right)=\mathcal{D}\left(P_{A, \min }\right) \oplus W^{-}$. By Theorem 4.4, $P_{A}^{i D}=P_{A, W^{-}}$and

$$
\operatorname{Ind} P_{A, W}=\operatorname{Ind} P_{A, \min }+\operatorname{dim} W=\operatorname{Ind} P_{A}^{i D}+\operatorname{dim} W-\operatorname{dim} W^{-} .
$$

Let $E_{s}(P)$ be the eigenspace of the operator $P$ with eigenvalue $s$. Then we have the following identification $(s \neq-1 / 2)$

$$
E_{s}\left(P_{0}\right)=E_{s+1 / 2, c e}^{1}\left(*_{Y} d_{a_{0}}\right) \oplus E_{\lambda(s)}^{0}\left(\Delta^{0}\right),
$$

where $E_{t, c c}^{1}\left(*_{Y} d_{a_{0}}\right)=\left\{\alpha_{1} \in \Omega^{1}: *_{Y} d_{a_{0}} \alpha_{1}=t \alpha_{1}, d_{a_{0}}^{*_{Y}} \alpha_{1}=0\right\}$. For $u=(\alpha, \phi) \in$ $E_{s}\left(P_{0}\right)$, define a map

$$
F(\alpha, \phi)=\left(\alpha-(s+1 / 2)^{-1} d_{a_{0}} \phi, \phi\right)
$$

The map $F$ is bijective since the equation $P_{0} u=s u$ is equivalent to $\Delta^{0} \phi=$ $\lambda(s) \phi$ and

$$
\begin{gathered}
*_{Y} d_{a_{0}}\left(\alpha-(s+1 / 2)^{-1} d_{a_{0}} \phi\right)=(s+1 / 2)\left(\alpha-(s+1 / 2)^{-1} d_{a_{0}} \phi\right), \\
d^{*_{Y}}\left(\alpha-(s+1 / 2)^{-1} d \phi\right)=0 .
\end{gathered}
$$

Note that for $s=-1 / 2, E_{-1 / 2}\left(P_{0}\right)=H^{1}\left(Y, \operatorname{ad} a_{0}\right) \oplus H^{0}\left(Y, \operatorname{ad} a_{0}\right)$ and $E_{3 / 2}\left(P_{0}\right)=$ $E_{2, c c}^{1}\left(*_{Y} d_{a_{0}}\right) \oplus H^{0}\left(Y, \operatorname{ad} a_{0}\right)$. For $-1 / 2<s<3 / 2, E_{s}\left(P_{0}\right) \cong H_{s+1 / 2, c c}^{1}\left(*_{Y} d_{a_{0}}\right)$. Let $m_{t}^{1}=\operatorname{dim} E_{t, c c}^{1}\left(*_{Y} d_{a_{0}}\right)$ and $m_{t}^{0}=\operatorname{dim} E_{t}^{0}\left(\Delta^{0}\right)$. Using the above discussion, 
one obtains

$$
\begin{aligned}
\eta_{P_{0}}(z)= & \sum_{s \in \operatorname{Spec} P_{0} \backslash\{0\}} \operatorname{sgn} s|s|^{-z} \\
= & -\sum_{s<0}+\sum_{s>0}|s|^{-z} \\
= & -\left(h^{1}+h^{0}\right) 2^{z}+h^{0}\left(\frac{3}{2}\right)^{-z}-\sum_{s<0, s \neq-1 / 2} m_{s+1 / 2}^{1}|s|^{-z} \\
& +\sum_{s>0} m_{s+1 / 2}^{1}|s|^{-z}-\sum_{s<-1 / 2} m_{\lambda(s)}^{0}|s|^{-z}+\sum_{s>3 / 2} m_{\lambda(s)}^{0}|s|^{-z} \\
= & -\left(h^{1}+h^{0}\right) 2^{z}+h^{0}\left(\frac{2}{3}\right)^{z}-2 \sum_{-1 / 2<s<0} m_{s+1 / 2}^{1}|s|^{-z} \\
& +\bar{\eta}_{*_{Y} d_{a_{0}}}(z)+\bar{\eta}_{\Delta^{0}}(z),
\end{aligned}
$$

where $h^{i}=\operatorname{dim} \operatorname{ker} \Delta^{i}$ and

$$
\begin{gathered}
\bar{\eta}_{*_{Y} d_{a_{0}}}(z)=\sum_{\lambda \in \operatorname{Spec}\left(*_{Y} d_{a_{0}}\right) \backslash\{0\}} \operatorname{sgn} \lambda\left|\lambda-\frac{1}{2}\right|^{-z}, \\
\bar{\eta}_{\Delta^{0}}(z)=\sum_{\lambda \in \operatorname{Spec} \Delta^{0} \backslash\{0\}} m_{\lambda}^{0}\left(\left|\frac{1}{2}+\sqrt{1+\lambda}\right|^{-z}-\left|\frac{1}{2}-\sqrt{1+\lambda}\right|^{-z}\right)
\end{gathered}
$$

from the equation $\lambda(s)=(s+1 / 2)(s-3 / 2)$. The more explicit calculation of the index will be discussed elsewhere.

Remark. Our eventual goal will be the study of gauge theory type of topological invariants for the conic 4-manifolds. Up to now, we focus on the analytic aspects of the linear theory. Deforming the metrics $d t^{2}+\left(t^{1-r}\right)^{2} g_{Y}$ with $0 \leq$ $r \leq 1$, the operator $P_{A}(r)$ changes from $P_{A}(0)$ which we have discussed in this paper to $P_{A}(1)=\frac{\partial}{\partial t}+\left(\begin{array}{cc}*_{Y} d_{a} & d_{a} \\ d_{a}^{*} & 0\end{array}\right)$ under the product metric. The closed extension $P_{A, W^{-}}(0)$ of $P_{A}(0)$ changes to $P_{A, W^{-}}$(1) with the corresponding domain $\left\{u=\sum_{s} u_{s}(t): s \in \operatorname{Spec}\left(P_{0}(1)\right), s<0\right\}$ for $P_{0}(1)=\left(\begin{array}{cc}*_{Y} d_{a} & d_{a} \\ d_{a}^{*} & 0\end{array}\right)$. This is precisely the Atiyah-Patodi-Singer global boundary condition for 4manifold $X$ with a boundary $Y$. The index formula is given by $\int_{X} \tilde{c}_{2}(\operatorname{ad} P)-$ $\frac{1}{2}\left(\eta_{P_{0}(1)}+\operatorname{dim} \operatorname{ker} P_{0}(1)\right)$ from $\operatorname{tr}\left(P_{A}^{*}(1) P_{A}(1)\right)-\operatorname{tr}\left(P_{A}(1) P_{A}^{*}(1)\right)$. The last term $\sum_{k \geq 1} \frac{2^{-2 k}}{2 k(2 k+1)} \operatorname{Res}_{1} \eta_{P_{0}}(2 k)$ in Theorem 5.1 vanishes since the $m=-1$ case has non-residue terms in the calculation.

Acknowledgment. The authors were partially supported by the U. S. National Science Foundation. 


\title{
References
}

[1] J. Brüning and R. Seeley, An Index theorem for first order regular singular operators, American J. Math. 110 (1988), no. 4, 650-711.

[2] __ Regular singular asymptotics, Adv. Math. 58 (1985), no. 2, 133-148.

[3] J. Cheeger, On the Hodge theory of Riemannian pseudomanifolds, Proc. Symp. Pure. Math. 36 (1980), 91-146.

[4] _._. Spectral geometry of singular Riemannian spaces, J. Differential Geom. 18 (1983), no. 4, 575-657.

[5] S. K. Donaldson and P. B. Kronheimer, The Geometry of Four-manifolds, Oxford Mathematical Monographs, Oxford University Press, Oxford, 1990.

[6] S. Donaldson and D. Sullivan, Quasiconformal 4-manifolds, Acta Math. 163 (1989), no. $3-4,181-252$.

[7] N. Dunford and J. Schwartz, Linear operators Part II : Sepctral theory, Sefl adjoint operators in Hilbert space, Interscience, New York 1963.

[8] J. Etnyre and K. Honda, On symplectic cobordisms, Math. Ann. 323 (2002), no. 1, 31-39.

[9] R. Gopakumar and C. Vafa, On the gauge theory/geometry correspondence, Adv. Theor. Math. Phys. 3 (1999), no. 5, 1415-1443.

[10] P. B. Kronheimer and T. S. Mrowka, Gauge theory for embedded surfaces, $I, I I$, Topology 32 (1993), no. 4, 773-826 and 34 (1995), no. 1, 37-97.

[11] M. Lesch, Operators of Fuchs Type, Conical Singularities, and Asymptotic Methods, Teubner-Texte zur Mathematik 136, Stuttgart, Leipzig 1997.

[12] J. Weidmann, Linear Operators in Hilbert Spaces, GTM 68, Springer-Verlag 1980.

\author{
WEIPING LI \\ DEPARTMENT OF MATHEMATICS \\ OKLAHOMA STATE UNIVERSITY \\ STILLWATER, OKLAHOMA \\ 74078-0613, U. S. A. \\ E-mail address: wli@math.okstate.edu \\ SHUGUANG WANG \\ Department of Mathematics \\ UNIVERSITY OF MISSOURI \\ Columbia, Missouri \\ 65211 U. S. A. \\ E-mail address: swOmath.missouri.edu
}

